\title{
Development of the Downhole Data Memory Releasing Device
}

\author{
Jibo $\mathrm{Li}^{1, \mathrm{a}^{*}}$, Weining $\mathrm{Ni}^{1, \mathrm{~b}}$ and Sanguo $\mathrm{Li}^{1, \mathrm{c}}$ \\ ${ }^{1}$ SINOPEC Research Institute of Petroleum Engineering, Beijing, 100101, China \\ alijb.sripe@sinopec.com, ${ }^{b}$ niwn.sripe@sinopec.com, ${ }^{c}$ lisg.sripe@sinopec.com
}

Keywords: LWD, RFID, mud circulation, annulus, memory

Abstract. With the development of geologic mapping and resource evaluation technology, traditional data transmission method, such as mud pulse telemetry and low-frequency electromagnetic telemetry, can't meet the requirement of large amount of LWD data transmission in downhole. This paper gives a new transmission method of LWD data by releasing radio frequency (RF) memories while drilling a well. A downhole sub available to releasing radio frequency memories is designed in this paper. Plenty of RF memories with LWD data are located in cavity of the releasing sub, and were released into the annulus regularly, which will return to the surface following mud circulation. In order to expand the storage capacity of the memories, wireless charging high capacity RF memories are designed, the capacity of which depends on the energy transferred from the RF reader in the releasing sub. At last, the memories are collected at the surface, and the data storied in them are read out and analyzed, which can be used to guide the drilling process.

\section{Introduction}

In oil-gas exploration and well drilling industry, data transmission from downhole to the surface is always restricting the development of MWD/LWD. Generally, cable logging is needed if you want to understand the situation of underground, in which multi-core cable was used to transmit logging data; this method is unsuitable in the process of drilling. In order to transmit the logging data to the surface rapidly in the process of drilling, most commonly used transmission methods are cable transmission and wireless transmission. Wireless transmission method includes mud pulse telemetry, low frequency electromagnetic telemetry and acoustic telemetry [1-4]. The representative method of cable transmission is intelligent drill string [5], but this method is seldom used due to technical difficulty and high cost of production services. The traditional mud pulse telemetry and electromagnetic wave transmission are the industrial standard of the measurement while drilling data acquisition, the typical transmission speed is 3 6bit/s, under the perfect condition can achieve $12 \mathrm{bit} / \mathrm{s}$, but this speed is too low for high resolution data acquisition and high density data work, such as the gamma imaging while drilling. By the method of releasing large capacity radio memory will be able to effectively solve the problem.

\section{Data transmission system}

The data transmission method while drilling contains three key technology, fast reading and writing technology of large capacity memory, high reliable releasing technology, LWD data collection and analysis on the surface. Fast reading and writing technology is used to read and write MWD/LWD data between the tools and radio frequency memories, high reliable releasing technology is used to release the memories with logging data into the annulus, LWD data collection and analysis technology is used to collect and analyze the data stored in the memories on the surface.

\section{Reading and writing of the RF memory}

In recent years, many research works have been carried out for the application of RFID technology in downhole data transmission [6], this paper gives a downhole data transmission system based on RFID technology in the analysis of this research work. 
The complete low frequency RFID system consists of three parts: RF micro memory, RF reader and remote data processing computer [7]. RF micro memory, also called RFID card, with the ability of intelligent read and write, and encrypted communication, including antenna, matching network, charging module, transmission module, storage module and so on. Low frequency reader consists of antenna, wireless matching module, reader chip and a microprocessor. A request signal was sent to the memory through modulated RF signal, the memory reply the identification information and then the reader sends signals to the computer or other data processing unit for further information processing.

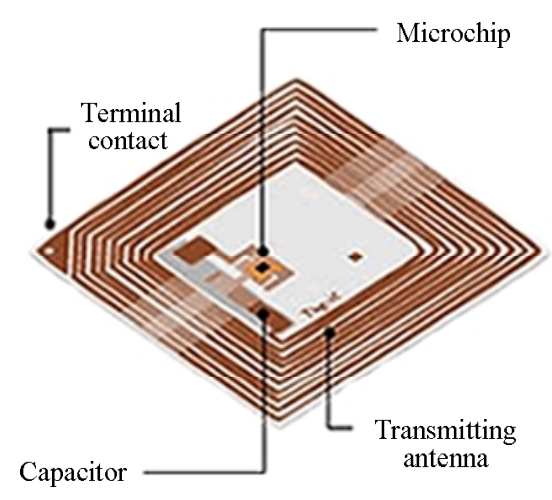

Fig.1 Traditional RFID Card

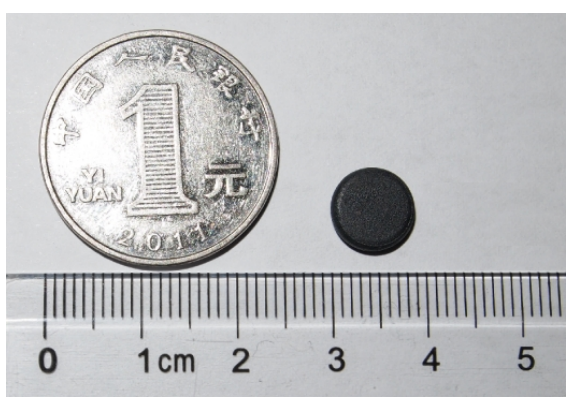

Fig.2 Prototype of large capacity RF memory

As shown in Fig. 1, RFID card usually made into traditional form, the size of the card is mainly determined by the size of the antenna. The card itself with wireless charging capabilities, at first was wirelessly charged, and then internal circuit started and completed identity recognition function. But for the application requirements, the capacity of the RF memory is relatively small, cannot meet the requirement of large data transmission while drilling in a well, so an external memory is needed to expand the storage capacity.

Because the RF memories have to work in high temperature, high pressure, and have to meet the requirements of life, temperature and repeated usage, wireless charging technology was used to solve these problems. The technology based on the wireless power transmission technology, also known as induction charging or non-contact inductive charging, is used in near field inductive power supply equipment. The device using the energy received to charge capacitor, and at the same time for its own use. Because energy transmission between the charger and the electrical device through coupled inductor, not by wires, so the charger and the electrical device do not have to be kept in contact. Fig. 2 is the prototype of large capacity memory using wireless charging technology.

Taking dimension into account, antenna becomes the most difficult part to design. Because the passive memory mainly works in hundreds of $\mathrm{KHZ}$ to several $\mathrm{MHz}$, the antenna of these frequency bands mainly depends on the coupling inductance. From Eq. 1 we can see that if the other conditions remain unchanged, the more turns of coil the greater of the magnetic field strength, the smaller of the radius the smaller of the magnetic field strength. Therefore, in the constraint of the dimension, the more turns of the coil the greater of the magnetic strength, which is better to the performance of the RF memory.

$$
H=\frac{I \cdot N \cdot R^{2}}{2\left(R^{2}+x^{2}\right)^{3}}
$$

Where, $H$ is the magnetic field strength, $I$ is current intensity, $N$ is the number of turns of the coil, $R$ is the radius of the antenna, $x$ is the distance.

In order to make the maximum emission efficiency, the resonant frequency must bring into correspondence with the working frequency, which is to satisfy Eq. 2.

$$
f=\frac{1}{2 \pi \sqrt{L C}}
$$

Where, $f$ is the working frequency, $C$ is the RF memory capacitor; $L$ is the inductance of the antenna. 


$$
L=\left(0.01 \times D \times N^{2}\right) /(l / D+0.44)
$$

Where, $L$ is the inductance of the coil, $D$ is the diameter of the coil, $N$ is the number of turns of the coil, $l$ is the length of the coil.

From Eq. 2 and Eq. 3, we can see that the turn number of the coil must in a certain range to make sure that the inductance of the coil $\mathrm{L}$ in the resonant frequency range. Combine Eq. 1, Eq. 2 and Eq. 3, for the memory of working frequency $13.56 \mathrm{MHz}$, the number of turns of the coil is selected as 50, and the diameter of the coupled antenna is selected as $8 \mathrm{~mm}$. For the memory of working frequency 134 $\mathrm{KHz}$, the number of turns of the coil is selected as 100, and the diameter of the coupled antenna is selected as $2 \mathrm{~mm}$.

\section{Memory releasing device}

The releasing sub is connected with other logging while drilling tools and the logging data from various while drilling logging tools are transferred to the memories through radio frequency method. There is a cavity in the while drilling releasing sub, one or more micro RF memories are placed in the cavity, and the logging data transferred from other logging tools are written into the memories through wireless transmission technology. The releasing sub regularly or irregularly releases the memories into the annulus, and then they will follow drilling fluid to the surface. The receiving device on the surface collects the memories. The memories do not have to go through the bit nozzle, so their dimensions can be larger and the communication efficiency is higher. The continuous releasing to the annulus can be dealt as quasi continuous data transmission.

The releasing sub comprises a signal receiving antenna, a storage module, a control module, data reading and writing antenna, a releasing actuator and a releasing hole. The signal receiving antenna is used to receive releasing instructions from the surface, the storage module is used to store the logging data, the control module is used to install control circuit board, the data reading and writing antenna is used to transfer logging data into the memories, the releasing actuator is used to provide releasing driven forces, and the releasing hole is used as the passageway of the memories from the storage module to the drilling fluid.

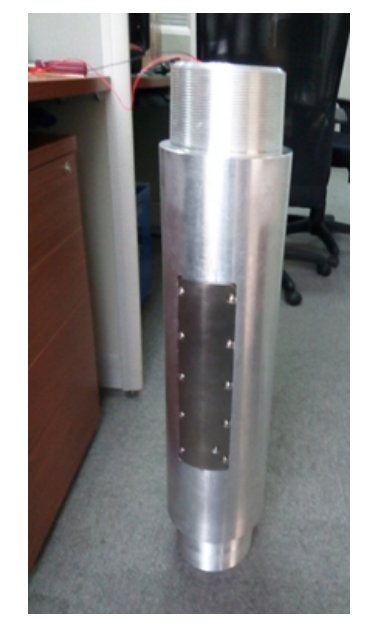

Fig.3 Physical map of the releasing sub

The main principle of the releasing sub is going to use the difference of the mud pressure inside and outside of the collar to provide the releasing force of the micro memory, the main working process is: when the logging data is needed on the surface, tags with releasing instructions are sent into the wellhead, the instruction tags are taken to the signal receiving antenna by the drilling fluid, and then the receiving antenna read the releasing instruction in the tag by RFID technology. The control circuit module transfers the logging data into the micro memory according to the instructions received from the receiving antenna. Releasing actuator control the releasing action of the memories after receiving instructions. Fig. 3 is the physical map of the releasing sub we designed. 


\section{Data collection and analysis on the surface}

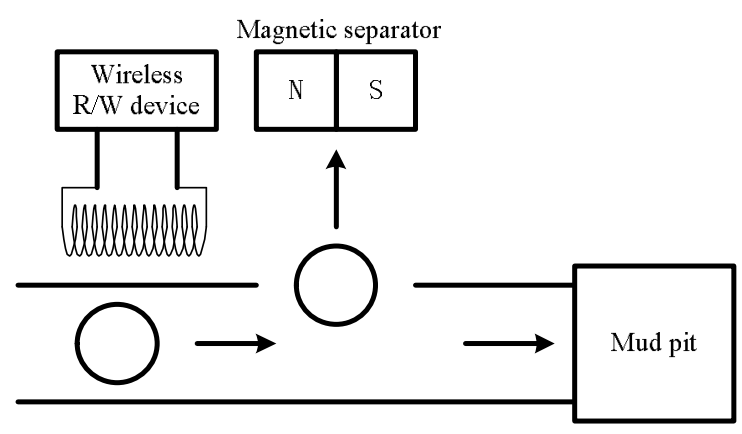

Fig. 4 Collection of the memories on the surface

Surface information processing system includes a data acquisition device and surface wireless reading device, monitor, see Fig. 4 . When the memories arrived at the surface, the data stored in them are read out, processed and displayed by the information processing system. The wireless reading device and magnetic separator are fixed near to the wellhead. The wireless reading device will read the logging data stored in the memories by RFID technology when the memories passed through, and the magnetic separator will catch the memories through the magnetic field because there are magnetic metal materials are contained in the memories.

\section{Summary}

Through the analysis, design and test above, downhole information transmission is feasible using RFID technology. Next step of our research will focus on the data processing and robust releasing test of the sub. High reliability requirements of the releasing sub in high temperature and high pressure environment of underground will also be the focus of our research work.

\section{References}

[1] X.S. Liu, Y.N. Su, Study on transmission velocity of mud pulse signal, Petroleum Drilling Technologies, 25 (2000) 24-26.

[2] X.P. Liu, J. Fang, Y.H. Jin, Application status and prospect of LWD data transmission technology, Well Logging Technology, 32(2008) 249-253.

[3] J. Zhu, Construction of the drilling fluid continuous pressure wave QPSK signals and its transmission characteristics study in the directional wellbore, China University of Petroleum (Hua Dong), 2010.

[4] C. Li, The research and development of MWD data acoustic transmission technology based on drill pipe, China University of Petroleum (Hua Dong), 2010.

[5] X.C. Liu, S.H. Zhang, Study on information and power transmission based on an intelligent drill string, Petroleum Drilling Technologies, 34(2006) 10-13.

[6] P.M. Sinder, Tom Doig, RFID Actuation of Self Powered Downhole Tools. SPE 2008.

[7] T.S. Song, J.L. Shen, Y.J. Qu, Design of digital logic circuit in a RFID transponder, Journal of Qingdao University of Science and Technology (Natural Science Edition), 29(2008) 72-76. 